\title{
ANALYSIS AND APPROXIMATION FOR INVERSE PROBLEMS IN CONTAMINANT TRANSPORT AND BIODEGRADATION MODELS*
}

\author{
Ben G. Fitzpatrick \\ Center for Research in Scientific Computation \\ and \\ Department of Mathematics \\ North Carolina State University \\ Raleigh, NC 27695-8205
}

\begin{abstract}
In this paper we consider the problem of estimating transport and biodegradation parameters in a contaminant transport model. We develop a convergence theory for parameter identification under approximation that includes the nonlinearities inherent in the biodegradation models. The functional analytic approach provides a means for studying a variety of solute transport and biodegradation problems.
\end{abstract}

*Research supported in part by AFOSR contract number F49620-93-1-0153. 
1. Introduction. Remediation of contaminated groundwater is a major scientific and technological challenge. One promising approach is bioremediation, in which either naturally occurring or externally introduced bacteria are used to decompose contaminants into non-toxic by-products. Important elements in the design of bioremediation strategies include mathematical models and computational algorithms for accurate prediction of contaminant degradation and transport. The literature on modeling and computation for biodegradation processes is quite extensive. We refer the reader to $[6,7,9,10,15,19,20]$, for discussion on model development and computational algorithms. In these works the primary focus is on computer simulation of biodegradation. In some cases, the simulations are compared to laboratory (see [20]) and field (see [7]) data for hydrocarbon degradation. In both cases, difficulties arise in the determination of parameter values for certain terms in the models. The goal of this paper is to develop a general inverse methodology for parameter estimation, comparing data to (computational approximations of) model based predictions in a least squares setting.

The paper is organized as follows. In Section 2, we describe the models and their parameters, and we indicate the typical inverse problems encountered in biodegradation data analysis. A functional-analytic theoretical framework for well-posedness and approximation is discussed in Section 3, and we connect our models to the abstract setting in Section 4. The convergence analysis for inverse problem is the topic of Section 5, and remarks and further studies are the topics of Section 6.

\section{Modeling and Inverse Problems in Contaminant Transport and Biodegra-}

dation. The basic physical phenomena of interest in contaminant transport through porous media are advection, dispersion, adsorption, and degradation. There are many possible system behaviors, and these depend heavily on the specific contaminants and 
soil types. We consider here transport of contaminants which dissolve into the groundwater. Basic assumptions include the following: the contaminants are at a sufficiently low level that their presence does not change the groundwater velocity; adsorption reactions are linear and occur at a much faster rate than the flow and dispersion; the only chemical reactions that change the contaminants are biodegradation reactions, which are enzyme-mediated; the transport is taking place within a medium which is saturated with groundwater. Mass-balance considerations lead to a model for a single solute of the form

$$
\begin{aligned}
\left(n(x)+R(x)(1-n(x)) c_{t}(t, x)\right. & +\nabla \cdot(v(x) c(t, x)) \\
& =\nabla \cdot(D(v(x)) \nabla c(t, x))-f(c(t, x), x),
\end{aligned}
$$

for $t>0, \quad x \in \Omega$, with initial condition $c(0, x)=c_{0}(x)$. In this model $c(t, x)$ is the contaminant concentration at time $t$, position $x, v=v(x)$ is the groundwater velocity vector, $D=D(v(x))$ is the dispersion matrix, $R(x)$ is the (linear) adsorption coefficient, $n(x)$ is the medium's porosity, and $f=f(c, x)$ is the rate at which the contaminant is degraded. The reader may consult $[4,12]$ for a full description of this model. Below, for completeness, we discuss briefly each of the terms.

The porosity $n$ represents the amount of volume which is not taken up by soil. For this quantity to be well-defined, one must choose a representative volume scale (see [4]). The soil, then takes up the remaining $1-n$ proportion of the volume. In saturated flow, $n c$ represents the volumetric concentration of the contaminant, while $c$ represents the concentration of contaminant in the water.

Adsorption is a process in which the solute is continually dissolving and adsorbing onto soil particles. A complete model of this phenomenon involves two phases, mobile and stationary. The linearity assumption is that this reaction occurs at a linear rate, 
and since the reaction occurs much faster than the flow, we take this reaction to be in equilibrium, so that the stationary phase concentration is proportional to the mobile phase concentration, leading to the given coefficient of the time derivative term. We set $\gamma=n+(1-n) R$. Sometimes this coefficient is referred to as the retardation factor.

The groundwater velocity, $v$, is a crucial component in our model. Typically, $v$ is modeled via Darcy's Law (see [4, 12]):

$$
v=-K \nabla h
$$

where $K$ is the hydraulic conductivity of the medium, and $h$ is the hydraulic head (the sum of the pressure head and elevation head). Mass conservation and incompressibility considerations lead to models of the form

$$
S h_{t}=\nabla \cdot K \nabla h, \quad \text { in } \Omega,
$$

with the equilibrium model being

$$
\nabla \cdot K \nabla h=0 \quad \text { in } \Omega
$$

In some circumstances one may wish to assume that $v$ has already been estimated and is known (problems of estimating $K$ have been considered in many works; see for example $[3,13,16])$. However, the estimation of $K$ is in itself a difficult matter and the topic of a great deal of research in geostatistics (see [11] for background and references). The results we obtain allow for the estimation of a steady state velocity field.

Another important feature of the model is the contaminant dispersion. The dispersion matrix $D$ takes the form

$$
D=d_{0} I+D_{1}(v)
$$


where $d_{0} I$ models molecular diffusion and $D_{1}(v)$ models the hydrodynamic dispersion of the solute that is due to the pore system of the medium and collisions of solute particles with soil particles. Empirical evidence and statistical considerations (see [4,Chap.10]) suggest the following model for $D_{1}$ :

$$
\left(D_{1}(v)\right)_{i j}=a_{T}|v| \delta_{i j}+\left(a_{L}-a_{T}\right) \frac{v_{i} v_{j}}{|v|} .
$$

Here $a_{T}$ is called the "transverse dispersivity," and $\alpha_{L}$ is called the "longitudinal dispersivity." In this dispersion model, the principal axis of dispersion is along the velocity vector (longitudinal), and there is less dispersion in directions orthogonal to $v\left(a_{T}<a_{L}\right)$. Moreover, the dispersion is modeled as (roughly) proportional to speed, the dispersion being caused by collisions with soil parrticles. See [4, Chap. 10] for a detailed exposition of dispersion.

The biodegradation term is commonly taken by hydrologists and engineers to be linear: $f(c)=\lambda c[\mathrm{Be}, \mathrm{FC}]$. Recently, however, more complex biological models have been employed $[6,7,9,10,15,19,20]$, such as the Monod or Michaelis-Menton/BriggsHaldane $[1,22,25]$ model of enzyme-mediated degradation, given by

$$
f(c)=\frac{\alpha c}{\beta+c} .
$$

Coupling the terms discussed above, we arrive at a model equation of the form

$$
\gamma(x) c_{t}+\sum_{i} \frac{\partial}{\partial x_{i}}\left(v_{i} c\right)=\sum_{i, j} \frac{\partial}{\partial x_{i}}\left(\left(a_{T}|v| \delta_{i j}+\left(a_{L}-a_{T}\right) \frac{v_{i} v_{j}}{|v|}\right) \frac{\partial c}{\partial x_{j}}\right)-f(c),
$$

with $D(v)$ given in $(3)$, and unknown parameters $c_{o}, \gamma, a_{L}, a_{T}$, and $f$ (which may or may not be parameterized as mentioned above) to be estimated.

In some cases, the biodegradation that takes place requires the presence of another nutrient, such as oxygen. The model given above can be considered as a case in which 
oxygen is abundant and the bacteria population is in steady state. A more realistic model would include a second differential equation for dissolved oxygen, and a third for the bacteria population. For example, in [10], the following system is employed:

$$
\begin{aligned}
\gamma_{c} c_{t}(t, x)+\nabla \cdot(v(x) c(t, x)) & =\nabla \cdot\left(D_{c}(v(x)) \nabla c(t, x)\right)-b K_{c} \frac{c}{\beta_{c}+c} \frac{o}{k_{o}+o}, \\
\gamma_{o} o_{t}(t, x)+\nabla \cdot(v(x) o(t, x)) & =\nabla \cdot\left(D_{o}(v(x)) \nabla o(t, x)\right)-b K_{o} \frac{c}{\beta_{c}+c} \frac{o}{k_{o}+o} \\
b_{t} & =b K_{b} \frac{c}{\beta_{c}+c} \frac{o}{k_{o}+o}-d b+B
\end{aligned}
$$

which involves contaminant concentration $c$, oxygen concentration $o$, and bacteria population $b$. The oxygen/contaminant reaction is assumed to obey a multiplicative Monod relationship, which is linear in bacteria population size. The bacteria are assumed to multiply at a rate proportional to their uptake of oxygen/contaminant, with a natural death rate $d$ and a constant birth rate $B$ which determines the "equilibrium" population size in the absence of nutrients. The true nature of the uptake of nutrients is still a topic of research in the biology and ecology literature, however. Recent analysis (see [23]) indicates that an additive Monod kinetic term of the form

$$
f(c, o)=\frac{k_{1} c o}{k_{2} c+c o+k_{3} o}
$$

provides a much better fit to batch reactor data than does a multiplicative model. In order to use models for field scale prediction and remediation aids, one must examine these biodegradation models within the context of dynamic lab and field scale experiments. The parameter estimation techniques presented here form the theoretical basis for such investigations.

In the models discussed above, some of the parameter values may be determined directly from experimental measurements, but others, most notably the degradation and dispersion terms, must be inferred from concentration data. Moreover, velocity informa- 
tion estimated from hydraulic head and conductivity observations may still contain some uncertainty. Thus, we are lead to study estimation based on contaminant concentration observations. A major difficulty is that in field situations, the medium is heterogeneous, which means the parameters to be estimated are spatially varying.

As an example, in the single solute transport model, data for the inverse problem is taken to be of the one of the following forms:

$$
\hat{C}_{i j}^{d} \approx \int_{\Omega_{j}} c\left(t_{i}, x\right) d x
$$

which denotes the dissolved contaminant level (mass as opposed to concentration) at time $t_{i}$ in the region $\Omega_{j}$;

$$
\hat{C}_{i j}^{t} \approx \int_{\Omega_{j}} \gamma(x) c\left(t_{i}, x\right) d x
$$

which denotes the total contaminant level (adsorbed and dissolved) at time $t_{i}$ in the region $\Omega_{j}$; or

$$
\hat{c}^{d}\left(t_{i}, x\right) \approx c\left(t_{i}, x\right)
$$

which denotes a complete dissolved contaminant image; or

$$
\hat{c}^{t}\left(t_{i}, x\right) \approx \gamma(x) c\left(t_{i}, x\right)
$$

which denotes a total (dissolved and adsorbed) image. The first two types of observations can be obtained from multilevel samplers, which are intrusive, well-type devices. The last two forms of observations are potentially available through nonintrusive, electromagnetic (e.g., ground penetrating radar) devices using dielectric constants to measure contaminant levels. Of course, producing such a "measurement" involves an inverse problem in electromagnetics, converting the radar signal to dielectric information. We will not examine this problem here: we merely wish to indicate that our theoretical and 
approximation studies provide a framework for analyzing the use of data produced by these sensors.

We shall consider a least squares estimation technique, based on the cost functional

$$
J\left(c_{o}, v, \gamma, a_{L}, a_{T}, f\right)=\left\|z-\mathcal{B} c\left(c_{o}, v, \gamma, a_{L}, a_{T}, f\right)\right\|_{Z}^{2}
$$

in which $Z$ denotes the measurement space (discrete or distributed), $z \in Z$ is one of the above types of data, $\mathcal{B}$ denotes the observation operator (whose possible forms are given above), and the parametric dependence of the concentration is explicitly noted. Again, in gerenal these parameters may be functions. The oxygen/contaminant/bacteria model involves similar measurements but considerably more parameters.

The problem of minimizing $J$ requires several steps. We must choose (i) a set of parameters over which we minimize, (ii) an approximation scheme for computing $c(t, x)$, and (iii) a numerical optimization technique for computing a minimizer. In order to perform these steps, we must understand the dependence of $c$ on the parameter vector $q=\left(c_{0}, v, \gamma, a_{L}, a_{T}, f\right)$. Further, we seek approximation methods which preserve the continuous dependence of solutions of the differential equations on parameters. Also, implementation of minimization methods requires a finite dimensional parameter space. In the next section, we consider an abstract framework for analyzing the dependence of solutions of equations such as (1), under approximation, on parameters. The theoretical results given below provide a means for structuring numerical parameter estimation algorithms in such a way as to obtain stable results with respect to perturbations in the data.

\section{Abstract Well-Posedness and Approximation Results.}

The abstract formulation and analysis of implicit, semilinear parabolic problems 
considered here relies on a combination of methods from the literature (see, in particular, $[2,21,24,26])$. Our treatment of the "implicitness" is a straightforward application of results in [26]: the more difficult degenerate problem is studied for linear problems in $[17,18]$. The nonlinearity of our problem can be studied using the theory given in [21, 24], and the approximation and parameter estimation results are derived by adapting the approach of [2] to handle the nonlinear term.

We begin with an abstract parameter dependent differential equation

$$
B(q) \dot{u}(t ; q)=A(q) u(t ; q)+F(t, u(t ; q) ; q)
$$

on a Hilbert space $H$, with parameter $q$ belonging to a compact separable metric space Q. This generality will allow us to examine most models of interest in bioremediation studies. We shall also assume that we have a Hilbert space $V$ which is densely and continuously embedded in $H$, for a Gelfand triple structure $V \hookrightarrow H=H^{*} \hookrightarrow V^{*}$. We remark that the "implicit" structure of equation (4) is motivated by our application, due to the adsorption term $\gamma$ (in the following section we shall discuss the connections between the abstract setting and the contaminant transport problem in detail). As we are assuming $\gamma>0$, we could divide through by $\gamma$ and solve the explicit problem, but at times it will be more convenient in the analysis to look directly at the implicit problem.

We now make some specific assumptions concerning the operators $A, B$ and $F$ in the equation (4).

(B1) There exist $b_{1} \geq b_{0}>0 \ni b_{0}^{2}|\phi|_{H}^{2} \leq\langle B(q) \phi, \phi\rangle \leq b_{1}^{2}|\phi|_{H}^{2}, \forall \phi \in H, q \in Q$; moreover, $B(q)$ is self-adjoint, and $B, B^{-1}$ are strongly continuous in $q$.

This assumption ensures that equation (4) is nondegenerate. The operator $A$ is assumed to be determined by a parameter dependent sesquilinear form on $V$; i.e., $\sigma(\cdot)(\cdot, \cdot)$ : 
$Q \times V \times V \rightarrow \mathbb{C}$, where $\sigma(q)(\cdot, \cdot)$ is sesquilinear for each $q \in Q$. Concerning $\sigma$, we assume

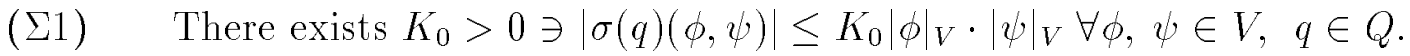

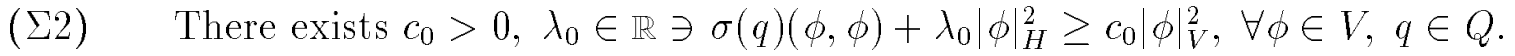

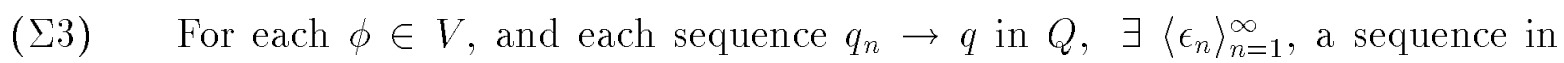
$\mathbb{R} \ni \epsilon_{n} \rightarrow 0$, with

$$
\left|\sigma(q)(\phi, \psi)-\sigma\left(q_{n}\right)(\phi, \psi)\right| \leq \epsilon_{n}|\psi|_{V}, \quad \forall \psi \in V
$$

Under these assumptions there exists a uniquely determined linear operator $A(q)$ : $\operatorname{dom}(A(q)) \rightarrow H$ satisfying $\sigma(q)(\phi, \psi)=\langle-A(q) \phi, \psi\rangle_{H}, \forall \phi \in \operatorname{dom} A(q), \psi \in V$.

For the nonlinear term, we shall need the following.

(F1) The function $F:[0, \infty) \times H \times Q \rightarrow H$ is continuous, and locally Lipschitz continuous in $H$, uniformly for $q \in Q$ and for $t$ in bounded intervals.

Under assumption (B1), we may invert $B(q)$ to obtain an explicit differential equation

$$
\begin{aligned}
\dot{u}(t ; q)= & B(q)^{-1} A(q) u(t ; q) \\
& +B(q)^{-1} F(t, u(t ; q) ; q) .
\end{aligned}
$$

In order to solve the semilinear equation (4) (equivalently $\left(4^{\prime}\right)$ ), we first seek a solution of the linear problem $\dot{u}=B^{-1} A u$. Toward that end, we define Hilbert spaces $\left\{H_{q}: q \in Q\right\}$, by considering $H$ endowed with the inner product $b(q)(\phi, \psi)=\langle B(q) \phi, \psi\rangle$. Assumption (B1) guarantees that $b(q)$ is a inner product on $H$, and that the $H$ and $H_{q}$ topologies are equivalent. Following [26,p.115] we consider the equation

$$
b(q)(\dot{u}, \phi)+\sigma(q)(u, \phi)=0, \quad \forall \phi \in V .
$$


From [26], we have the existence of an operator $L(q): \operatorname{dom}(L(q)) \rightarrow H_{q} \ni \sigma(q)(\phi, \psi)=$ $b(q)(-L(q) \phi, \psi)$, and that $L(q)$ is the infinitesimal generator of a $C_{0}$-semigroup $T(t ; q)$ on $H_{q}$ satisfying $|T(t ; q) \phi|_{H_{q}} \leq e^{\lambda_{0} t}|\phi|_{H_{q}}$, which in turn implies that $T(t ; q)$ is a $C_{0^{-}}$ semigroup on $H$ satisfying $|T(t ; q) \phi|_{H} \leq\left(b_{1} / b_{0}\right) e^{\lambda_{0} t}|\phi|_{H}$, through (B1). We may now consider the mild form of $\left(4^{\prime}\right)$, given by

$$
u(t ; q)=T(t ; q) u_{0}+\int_{0}^{t} T(t-s ; q) B^{-1}(q) F(s, u(s ; q) ; q) d s
$$

As can be seen in [21, Chap. 8, 24, Chap 6], equation (5) has a unique solution $u \in$ $C([0, \tau], H)$ for some $\tau>0$. If (F1) can be strenghtened to global Lipschitz, then we can take $\tau=\infty$. One can compute a lower bound for $\tau$, as in [24, pp. 184-187]: if $\left|u_{0}\right| \leq C_{0}$, then one has

$$
\tau \geq \tau_{0}=\min \left\{1, \frac{C_{0}}{\hat{K}_{0} L_{0} M_{0}+N_{0}}\right\},
$$

where

$$
\hat{K}_{0}=2 C_{0}\left(b_{1} / b_{0}\right) e^{\lambda_{0}} \geq 2 C_{0} \max \{|T(t ; q)|: q \in Q, t \in[0,1]\},
$$

$L_{0}$ is the local Lipschitz constant for $F$ associated with the ball $|x| \leq \hat{K}_{0}$ (for $t \in$ $[0,1], q \in Q), M_{0}=\sup _{q}\left|B^{-1}(q)\right|$, and $N_{0}=\max \left\{\left|B^{-1}(q) F(t, 0 ; q)\right|: t \in[0,1], q \in Q\right\}$. We also note that the mapping $\mathcal{F}_{u_{0}, q}: C\left(\left[0, \tau_{0}\right], H\right) \rightarrow C\left(\left[0, \tau_{0}\right], H\right)$ given by

$$
\mathcal{F}_{u_{0}, q}(u)(t)=T(t ; q) u_{0}+\int_{0}^{t} T(t-s ; q) B^{-1}(q) F(s, u(s) ; q) d s
$$

leaves the ball radius $\hat{K}_{0}$ invariant and is a contraction on that ball (moreover, the contraction is uniform) for $\left|u_{0}\right| \leq C$ and $q \in Q$. These facts (see [24, pp. 184-187] for details) will be required also for our convergence analysis.

Next, we consider an approximation method based on a sequence of Hilbert spaces $H^{N}, N=1,2, \ldots$, with orthogonal projections $P^{N}: H \rightarrow H^{N}$. The following assumption about these approximations will be needed for our convergence results. 
(A1) The subspaces $H^{N}$ are subsets of $V$, and $\forall v \in V, \exists v^{N} \in H^{N} \ni\left|v^{N}-v\right|_{V} \rightarrow 0$.

This assumption is satisfied by many finite element and spectral schemes (see [3, 8, 14]). The Galerkin approach to approximation involves restricting $b(q)$ and $\sigma(q)$ to $H^{N} \times H^{N}$, yielding bounded linear operators $A^{N}(q), L^{N}(q)$ satisfying

$$
\begin{aligned}
\sigma(q)\left(\phi^{N}, \psi^{N}\right) & =-\left\langle A^{N}(q) \phi^{N}, \psi^{N}\right\rangle \\
& =-b(q)\left(L^{N}(q) \phi^{N}, \psi^{N}\right) .
\end{aligned}
$$

Now, $\forall q, L^{N}(q)$ generates a $C_{0}$-semigroup $T^{N}(t ; q)$ on $H^{N}$ satisfying

$$
\left|T^{N}(t ; q)\right|_{\mathcal{L}\left(H^{N}\right)} \leq\left(b_{1} / b_{0}\right) e^{\lambda_{0} t}
$$

where $\mathcal{L}\left(H^{N}\right)$ is endowed with the topology inherited from $H$. Since

$$
\left\langle A^{N}(q) \phi^{N}, \psi^{N}\right\rangle=\left\langle B(q) L^{N}(q) \phi^{N}, \psi^{N}\right\rangle \quad \forall \phi^{N}, \psi^{N} \in H^{N}
$$

we have

$$
A^{N}(q) \phi^{N}=P^{N} B(q) L^{N}(q) \phi^{N} \quad \forall \phi^{N} \in H^{N}
$$

Now, the strong and mild forms for the approximate equations are given by

$$
\dot{u}^{N}(t)=L^{N}(q) u^{N}+P^{N} B^{-1}(q) F\left(t ; u^{N} ; q\right)
$$

and

$$
u^{N}(t)=T^{N}(t ; q) P^{N} u_{0}+\int_{0}^{t} T^{N}(t-s ; q) P^{N} B^{-1}(q) F\left(s, u^{N}(s) ; q\right) d s
$$

We remark that (7) has a unique mild solution (as above) and that the maximum interval of existence estimate will be the same under the above stability condition on the semigroup, the boundedness of the projections $P^{N}$, and the continuity of $B$.

Within the context of inverse problems, we seek to show that $u^{N}\left(t ; q^{N}\right) \rightarrow u(t ; q)$ as $q^{N} \rightarrow q$, which is a crucial step in parameter identification (see [2, 3]). We first consider the linear part of the equation. The following result, known as the Trotter-Kato theorem (see, e.g. $[2,3,24]$ ), will be at the heart of our analysis. 
Theorem 0. Let $A^{N}$ and $A$ be infinitesimal generators of $C_{0}$-semigroups $S^{N}(t)$ and $S(t)$ on Hilbert spaces $X^{N}$ and $X$, with $X^{N} \subset X$, and orthogonal projections $\mathcal{P}^{N}: X \rightarrow$ $X^{N}$. Suppose the following two conditions hold:

(i) $\quad \exists M, w \ni\left|S^{N}(t)\right|_{\mathcal{L}\left(X^{N}\right)} \leq M e^{\omega t}$, and

(ii) $\quad \exists \lambda \in \rho(A) \cap\left\{\cap_{N=1}^{\infty} \rho\left(A^{N}\right)\right\}$ with Re $\lambda>\omega$ and $R_{\lambda}\left(A^{N}\right) \mathcal{P}^{N} \xi \rightarrow R_{\lambda}(A) \xi \quad \forall \xi \in$ $H$.

Then $\mathcal{S}^{N}(t) \mathcal{P}^{N} x \rightarrow \mathcal{S}(t) x$, uniformly on each interval [0, $\left.\tau\right], \forall x \in X$.

Above we have verified (i) for the semigroups $T^{N}(t ; q)$, with bounds that are independent of $q \in Q$. Below, we verify the resolvent convergence condition (ii), which, in view of the Trotter-Kato theorem leads us to the following.

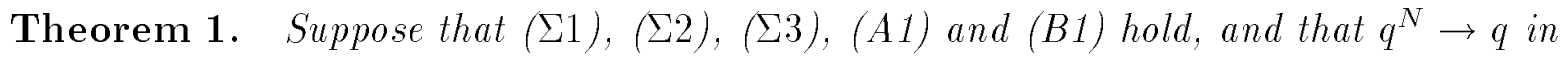
Q. Then we have that

$$
T^{N}\left(t ; q^{N}\right) P^{N} z \rightarrow T(t ; q) z
$$

uniformly on each interval $[0, \tau], \forall z \in H$.

Proof. Condition (ii) of Theorem 0 remains to be verified. Toward that end, we let $z \in H, \lambda>\lambda_{0}$. Note that, $\forall \phi \in \operatorname{dom}(A(q))$,

$$
\begin{aligned}
& \lambda\langle B(\tilde{q}) \phi, \phi\rangle+\sigma(q)(\phi, \phi) \\
& =\langle(\lambda B(\tilde{q})-A(\tilde{q})) \phi, \phi\rangle \geq c_{0}|\phi|_{V}^{2},
\end{aligned}
$$

so that $(\lambda B(q)-A(q))^{-1} \in \mathcal{L}(H)$. Likewise, by restricting attention to $H^{N}$, we have that $\left(\lambda B\left(q^{N}\right)-A^{N}\left(q^{N}\right)\right)^{-1} \in \mathcal{L}\left(H^{N}\right)$. Next, we set $w^{N}=\left(\lambda B^{N}\left(q^{N}\right)-A^{N}\left(q^{N}\right)\right)^{-1} P^{N} z$, with 
$B^{N}\left(q^{N}\right)=P^{N} B\left(q^{N}\right), w=(\lambda B(q)-A(q))^{-1} z$, and we choose $\hat{w}^{N} \in V \ni\left|\hat{w}^{N}-w\right|_{V} \rightarrow 0$. We set $z^{N}=w^{N}-\hat{w}^{N}$. We first show that $z^{N} \rightarrow 0$ in $V$. First, note that

$$
\begin{aligned}
\sigma(q)\left(w, z^{N}\right) & =\left\langle-A(q) w, z^{N}\right\rangle \\
& =\left\langle z, z^{N}\right\rangle-\lambda\left\langle B(q) w, z^{N}\right\rangle, \text { and } \\
\sigma\left(q^{N}\right)\left(w^{N}, z^{N}\right) & =\left\langle-A^{N}\left(q^{N}\right) w^{N}, z^{N}\right\rangle \\
& =\left\langle\left(\lambda B^{N}\left(q^{N}\right)-A^{N}\left(q^{N}\right)\right) w^{N}, z^{N}\right\rangle-\lambda\left\langle B^{N}\left(q^{N}\right) w^{N}, z^{N}\right\rangle \\
& =\left\langle P^{N} z, z^{N}\right\rangle-\lambda\left\langle B^{N}\left(q^{N}\right) w^{N}, z^{N}\right\rangle \\
& =\left\langle z, z^{N}\right\rangle-\lambda\left\langle B^{N}\left(q^{N}\right) w^{N}, z^{N}\right\rangle,
\end{aligned}
$$

so that

$$
\sigma(q)\left(w, z^{N}\right)=\sigma\left(q^{N}\right)\left(w^{N}, z^{N}\right)+\lambda\left(\left\langle B^{N}\left(q^{N}\right) w^{N}-B(q) w, z^{N}\right\rangle\right)
$$

Next, we have that

$$
\begin{aligned}
& c_{0}\left|z^{N}\right|_{V}^{2} \leq \sigma\left(q^{N}\right)\left(z^{N}, z^{N}\right)+\lambda\left|z_{N}\right|^{2} \\
& =\sigma\left(q^{N}\right)\left(w^{N}, z^{N}\right)-\sigma\left(q^{N}\right)\left(\hat{w}^{N}, z^{N}\right)+\lambda\left|z_{N}\right|^{2} \\
& =\sigma(q)\left(w, z^{N}\right)-\lambda\left\langle\left(B^{N}\left(q^{N}\right) w^{N}-B(q) w\right), z^{N}\right\rangle \\
& -\sigma\left(q^{N}\right)\left(\hat{w}^{N}, z^{N}\right)+\lambda\left|z^{N}\right|^{2} \\
& =\sigma(q)\left(w, z^{N}\right)-\sigma\left(q^{N}\right)\left(w, z^{N}\right) \\
& +\sigma\left(q^{N}\right)\left(w-\hat{w}^{N}, z^{N}\right)+\lambda\left|z_{N}\right|^{2} \\
& -\lambda\left\langle\left(B^{N}\left(q^{N}\right) w^{N}-B(q) w\right), z^{N}\right\rangle \\
& \leq \epsilon^{N}\left|z^{N}\right|_{V}+K_{0}\left|w-\hat{w}^{N}\right|_{V}\left|z^{N}\right|_{V} \\
& +\lambda\left|z_{N}\right|^{2}-\lambda\left\langle B^{N}\left(q^{N}\right) w^{N}-B(q) w, z^{N}\right\rangle \\
& =\epsilon^{N}\left|z^{N}\right|_{V}+K_{0}\left|w-\hat{w}^{N}\right|_{V}\left|z^{N}\right|_{V}
\end{aligned}
$$




$$
\begin{gathered}
+\lambda\left|z_{N}\right|^{2}-\lambda\left\langle B^{N}\left(q^{N}\right) w^{N}-B^{N}\left(q^{N}\right) \hat{w}^{N}, z^{N}\right\rangle \\
-\lambda\left\langle B^{N}\left(q^{N}\right) \hat{w}^{N}-B(q) w, z^{N}\right\rangle \\
\leq \epsilon^{N}\left|z^{N}\right|_{V}+\left.K_{0}\left|w-\hat{w}^{N}\right|_{V}\right|_{\left.z_{N}\right|_{V}} \\
+\lambda\left|z_{N}\right|_{H}^{2}-\lambda\left\langle B^{N}\left(q^{N}\right) z_{N}, z_{N}\right\rangle \\
-|\lambda|\left|B^{N}\left(q^{N}\right) \hat{w}^{N}-B(q) w\right|_{H}\left|z_{N}\right|_{H} \\
\leq \epsilon^{N}\left|z^{N}\right|_{V}+K_{0}\left|w-\hat{w}^{N}\right|_{V}\left|z_{N}\right|_{V} \\
+|\lambda|\left|B^{N}\left(q^{N}\right) \hat{w}^{N}-B(q) w\right|_{H} \cdot\left|z_{N}\right|_{H} .
\end{gathered}
$$

In the above, the sequence $\epsilon^{N}$ is chosen with respect to $w$. Thus, we have

$$
\begin{aligned}
c_{0}\left|z^{N}\right|_{V} \leq & \epsilon^{N}+K_{0}\left|w-\hat{w}^{N}\right|_{V} \\
& +|\lambda| k\left|B^{N}\left(q^{N}\right) \hat{w}^{N}-B(q) w\right|_{H},
\end{aligned}
$$

where $k$ is the imbedding constant $V \hookrightarrow H$ in $V$. Since $\epsilon^{N} \rightarrow 0,\left|w-\hat{w}^{N}\right|_{V}$ and $B$ is strongly continuous, we have that $z^{N} \rightarrow 0$ in $V$, due to the fact that if $\left\{C_{n}\right\}$ is a sequence of bounded linear operators that converges strongly to $C$, then $C_{n} x_{n} \rightarrow C x$ when $x_{n} \rightarrow x$.

Next, we note that

$$
\left(\lambda I-B^{-1}(q) A(q)\right)^{-1}=\left(B^{-1}(q)(\lambda B(q)-A(q))\right)^{-1}=(\lambda B(q)-A(q))^{-1} B(q),
$$

and that if

$$
\left(\lambda I-L^{N}\left(q^{N}\right)\right)^{-1} \phi^{N}=\psi^{N}
$$

then

$$
\phi^{N}=\left(\lambda I-L^{N}\left(q^{N}\right)\right) \psi^{N}
$$

so

$$
P^{N} B\left(q^{N}\right) \phi^{N}=\left(\lambda P^{N} B\left(q^{N}\right)-A^{N}\left(q^{N}\right)\right) \psi^{N},
$$


or

$$
\left(\lambda B^{N}\left(q^{N}\right)-A^{N}\left(q^{N}\right)\right)^{-1} P^{N} B\left(q^{N}\right) \phi^{N}=\psi^{N}
$$

Thus, for $z \in H$,

$$
\begin{aligned}
&\left(\lambda I-L^{N}\left(q^{N}\right)\right)^{-1} P^{N} z \\
&=\left(\lambda B^{N}\left(q^{N}\right)-A^{N}\left(q^{N}\right)\right)^{-1} P^{N}\left(B\left(q^{N}\right) P^{N} z\right) \\
& \rightarrow(\lambda B(q)-A(q))^{-1} B(q) z, \text { in } H,
\end{aligned}
$$

and we have the result.

We now let $u^{N}$ denote the solution of (7) with parameter $q^{N}$, initial condition $u_{0}^{N} \in$ $H^{N}$. We also denote by $u$ the solution of (5) with parameter $q$ and initial condition $u_{0}$. We further assume that $q^{N} \rightarrow q$ and that $u_{0}^{N} \rightarrow u_{0}$ in $H$. Note that a convergent sequence is bounded, so there is a number $C_{0}$ such that $\left|u_{0}\right|,\left|u_{0}^{N}\right| \leq C_{0}$, for all $N$. Choose $\hat{K}_{0}, L_{0}, M_{0}, N_{0}$, and $\tau_{0}$ for this value of $C_{0}$.

Next, we set $T^{N}(t)=T^{N}\left(t ; q^{N}\right)$ and $T(t)=T(t ; q)$. Comparing $(5)$ and $(7)$, we have that

$$
\begin{aligned}
& \left|u^{N}(t)-u(t)\right| \leq\left|T^{N}(t) u_{0}^{N}+T(t) u_{0}\right| \\
& \quad+\int_{0}^{t}\left|T^{N}(t-s) P^{N} B^{-1}\left(q^{N}\right) F\left(s, u^{N} ; q^{N}\right)-T(t-s) B^{-1}\left(q^{N}\right) F(s, u ; q)\right| d s .
\end{aligned}
$$

In view of the previous theorem, we need only concern ourselves with the second term.

Suppose that $0<\hat{\tau}<\tau_{0}$, that $t \in[0, \hat{\tau}], \tau_{0}$ being as defined above. Then

$$
\begin{aligned}
& \int_{0}^{t}\left|T^{N}(t-s) P^{N} B^{-1}\left(q^{N}\right) F\left(s, u^{N} ; q^{N}\right)-T(t-s) B^{-1}(q) F(s, u ; q)\right| d s \\
& \leq \int_{0}^{t}\left|T^{N}(t-s) P^{N} B^{-1}\left(q^{N}\right)\left(F\left(s, u^{N} ; q^{N}\right)-F\left(s, u ; q^{N}\right)\right)\right| d s \\
& +\int_{0}^{t}\left|T^{N}(t-s) P^{N}\left(B^{-1}\left(q^{N}\right) F\left(s, u^{N} ; q^{N}\right)-B^{-1}(q) F\left(s, u ; q^{N}\right)\right)\right| d s
\end{aligned}
$$




$$
\begin{aligned}
& +\int_{0}^{t}\left|T^{N}(t-s) P^{N} B^{-1}\left(q^{N}\right) F\left(s, u^{N} ; q^{N}\right)-T(t-s) B^{-1} F\left(s, u ; q^{N}\right)\right| d s \\
& \leq M \int_{0}^{t}\left|u^{N}(s)-u(s)\right| d s \\
& +M^{\prime} \int_{0}^{t}\left|B^{-1}\left(q^{N}\right) F\left(s, u ; q^{N}\right)-B^{-1}(q) F(s, u ; q)\right| d s \\
& +\int_{0}^{t} T^{N}(t-s) P^{N} B^{-1}(q) F(s, u ; q)-T(t-s) B^{-1}(q) F(s, u ; q) \mid d s,
\end{aligned}
$$

where $M, M^{\prime}$ depend on the bounds for $B^{-1},\left\{P^{N}\right\}_{N=1}^{\infty}, T(\cdot)$, and $M$ depends on the local Lipschitz constant for $F$ based (as mentioned above) on the ball of radius $\hat{K}_{0}$ in $H$. It is important to note that the solutions are bounded by $\hat{K}_{0}$ in $C\left(\left[0, \tau_{0}\right], H\right)$ uniformly in $N$, due to the uniform boundedness of the semigroups. For this reason, the local Lipschitz constant for $F$ may be used above.

Hence we have that

$$
\left|u^{N}(t)-u(t)\right| \leq \alpha^{N}(t)+\beta \int_{0}^{t}\left|u^{N}(s)-u(s)\right| d s,
$$

with $\alpha^{N}(t) \rightarrow 0$ as $N \rightarrow \infty$. A straightforward application of Gronwall's inequality, coupled with the fact that the length $\tau$ of the maximum interval of existence is bounded below - independently of $N$ - for the approximate problems, gives us the following.

Theorem 2. Assume that ( $\Sigma 1),(\Sigma 2),(\Sigma 3),(A 1),(B 1)$ and (F1) hold. Then, if $q^{N} \rightarrow q$ in $Q$, and $u_{0}^{N} \rightarrow u_{0}$ in $H$, we have that

$$
u^{N}\left(t ; q^{N}, u_{0}^{N}\right) \rightarrow u\left(t ; q, u_{0}\right) \quad \text { uniformly on each interval }[0, \hat{\tau}]
$$

in $H$, where $\hat{\tau} \leq \tau_{0}$.

Before proceeding to the inverse problem, we relate these abstract results to the contaminant transport problem of interest.

\section{Abstract Formulation of the Transport Equation.}


We begin with the Hilbert spaces $H=L^{2}(\Omega)$ and $V=H^{1}(\Omega)$, which are the usual Lebesgue and Sobolev spaces. We define the sesquilinear form $\sigma$ by

$$
\sigma(q)(\phi, \psi)=\int_{\Omega}(D(v) \nabla \phi \cdot \nabla \psi-v \phi \cdot \nabla \psi) d x
$$

which, when integrated by parts (using the no-flux boundary conditions), yields the convection and dispersion terms. We also define

$$
b(q)(\phi, \psi)=\int_{\Omega} \gamma(x) \phi(x) \psi(x) d x
$$

Here we have $q=\left(v, \gamma, a_{L}, a_{T}, f\right)$. The nonlinear term $F$ is given by $F(s, u ; q)=f(u(s))$. For our abstract formulation, we have

$$
b(q)(\dot{c}, \phi)+\sigma(q)(c, \phi)=\langle f(c), \phi\rangle
$$

where $\langle$,$\rangle is the usual L^{2}(\Omega)$ inner product. To satisfy the requirements of the previous section, we will need to make some restrictions on the parameter $q$. We set

$$
\tilde{Q}=\left(L^{2}(\Omega)\right)^{3} \times L^{\infty}(\Omega) \times\left(L^{\infty}(\Omega)\right)^{2} \times \operatorname{BUC}(\mathbb{R})
$$

where $L^{\infty}(\Omega)$ denotes the usual space of essentially bounded measurable functions, and $\mathrm{BUC}(\mathbb{R})$ denotes the space of bounded, uniformly continuous functions on $\mathbb{R}$ (which is a Banach space, with the sup norm). We next define a compact subset of $\widetilde{Q}$ in order to obtain a parameter set which will fulfill the conditions of the previous section. Let

$$
\begin{aligned}
& \mathcal{V}=\left\{v \in\left[L^{2}(\Omega)\right]^{3}:|v(x)| \leq K_{1} \text { a.e., } T V(v) \leq K_{2}\right\}, \\
& \Gamma=\left\{\gamma \in C^{0,1}(\bar{\Omega}): \gamma_{0} \leq \gamma,|\gamma|_{C^{0,1}} \leq K_{3}\right\} \\
& \mathcal{A}=\left\{\left(a_{L}, a_{T}\right) \in\left(C^{0,1}(\bar{\Omega})\right)^{2}: 0 \leq a_{T} \leq a_{L}\left|a_{L}\right|_{C^{0,1}} \leq K_{4}, \quad\left|a_{T}\right|_{C^{0,1}} \leq K_{5}\right\} \\
& \mathcal{F}=\left\{f \in C^{0,1}(\mathbb{R}): f(x)=0 \forall x \leq 0,|f|_{C^{0,1}} \leq K_{6}\right\} .
\end{aligned}
$$


An alternative for $\mathcal{F}$ incorporating the Michaelis-Menton model is given by

$$
\mathcal{F}_{m m}=\left\{f(c)=\max \left\{\frac{\alpha c}{\beta+c}, 0\right\}: \alpha, \beta \geq 0, \alpha, \beta \in C^{0,1}(\bar{\Omega}),|\alpha|_{C^{0,1}},|\beta|_{C^{0,1}} \leq K_{6}\right\}
$$

Here $\left\{\gamma_{0}, K_{1}, \ldots, K_{6}\right\}$ is a set of fixed positive constants. Also $T V(v)=\max _{1 \leq i \leq 3}\left\{T V\left(v_{i}\right)\right\}$, where $T V(f)=\sup \left\{\int_{\Omega} f(x) \operatorname{div}(\phi(x)) d x:|\phi(x)| \leq 1, \phi, \phi^{\prime} \in C(\bar{\Omega})\right\}$ is the total variation of $f$. The space $C^{0,1}(\bar{\Omega})$ is the space of Lipschitz continuous functions with norm

$$
|f|_{C^{0,1}}=\sup _{\Omega}|f(x)|+\sup _{\substack{x, y \in \Omega \\ x \neq y}}\left|\frac{f(x)-f(y)}{x-y}\right| \text {. }
$$

The set $Q=\mathcal{V} \times \Gamma \times \mathcal{A} \times \mathcal{F}$ is easily seen to be compact in $\widetilde{Q}$, using Arzela-Ascoli and Solobev imbeddings, coupled with compactness results in $B V$ such as are contained in [13]. We remark that there are many other possible choices for the set $Q$; the analysis, however, is quite similar for the different choices.

The assumptions $(\Sigma 1)$ and $(\Sigma 2)$ are easily verified using standard PDE arguments (see, e.g., [2]), and the fact that $d_{0}>0$ is known and fixed. To examine $(\Sigma 3)$, we let $\phi, \psi \in V$, and we suppose $q^{N} \rightarrow q$ in $Q$. We put

$$
D^{N}\left(v^{N}\right)_{i j}=d_{0} \delta_{i j}+a_{T}^{N}\left|v^{N}\right| \delta_{i j}+\left(a_{L}^{N}-a_{T}^{N}\right) \frac{v_{i}^{N} v_{j}^{N}}{\left|v^{N}\right|} .
$$

Then we have that

$$
\begin{aligned}
& \left|\sigma(q)(\phi, \psi)-\sigma\left(q^{N}\right)(\phi, \psi)\right| \\
& \leq \int_{\Omega}\left|D(v) \nabla \phi \cdot \nabla \psi-D^{N}\left(v^{N}\right) \nabla \phi \cdot \nabla \psi\right| d x \\
& +\int_{\Omega}\left|v^{N}-v\right||\phi| \cdot|\nabla \psi| d x \\
& \leq\left[\int_{\Omega}\left|D(v)-D^{N}\left(v^{N}\right)\right|^{2}|\nabla \phi|^{2} d x\right]^{1 / 2}|\psi|_{H^{1}} \\
& +\left[\int_{\Omega}\left|v^{N}-v\right||\phi|^{2} d x\right]^{1 / 2}|\psi|_{H^{1}} \\
& =\epsilon_{N}|\psi|_{H^{1}} .
\end{aligned}
$$


Since $v^{N} \rightarrow v$ in $L^{2}(\Omega), \quad\left|v^{N}(x)\right| \leq K_{1}, \quad a_{L}^{N} \rightarrow a_{L}, \quad a_{T}^{N} \rightarrow a_{T}$ in $L^{\infty}$, the dominated convergence theorem applies (as in [13]), and $\epsilon_{N} \rightarrow 0$.

Concerning $(\mathrm{F} 1)$, we have $F(t, c ; q)=f(c)$. By the definition of $\mathcal{F}$, the hypotheses of (F1) are easily seen to hold. In fact, this function is globally Lipschitz continuous, and the arguments of the previous section may be extended to compact but arbitrarily large time intervals.

In order to satisfy (A1), we may take a standard Galerkin finite element method (see $[3,14])$, such as $H^{N}=\operatorname{span}\left\{B_{1}^{N}, \ldots, B_{k_{N}}^{N}\right\}$, with $B_{i}^{N}$ being the tensor product of linear spline functions or linear functions on a triangulation of $\Omega$.

Finally, we have that

$$
b_{0}^{2} \int_{\Omega}|\phi|^{2} d x \leq \int_{\Omega} \gamma(x)|\phi(x)|^{2} \leq b_{1}^{2} \int_{\Omega}|\phi|^{2}
$$

where $b_{1}^{2}=K_{4}$ and $b_{0}^{2}=\gamma_{0}$. Also, we have $(B(q) \phi)(x)=(\gamma(x)) \phi(x)$, and if $q^{N} \rightarrow q$ in $Q$ so that $\gamma^{N} \rightarrow \gamma$ in $L^{\infty}$, we have $B\left(q^{N}\right) \phi \rightarrow B(q) \phi$. Likewise $\left(B^{-1}(q) \psi\right)(x)=\frac{1}{\gamma(x)} \psi(x)$ is strongly continuous in $q$, since $\gamma$ is uniformly bounded below by $\gamma_{0}>0$.

Having verified the conditions of Theorem 2, we have that $q^{N} \rightarrow q$ in $Q$ and $u_{0}^{N} \rightarrow u_{0}$ in $H$ implies $u^{N}\left(t ; q^{N}, u_{0}^{N}\right) \rightarrow u\left(t ; q, u_{0}\right)$ in $H=L^{2}(\Omega)$.

In the case of the oxygen limited reactions simulated in $[6,7,9,10,15,19,20]$, we have the system of equations

$$
\begin{aligned}
\gamma_{c} c_{t}(t, x)+\nabla \cdot(v(x) c(t, x)) & =\nabla \cdot\left(D_{c}(v(x)) \nabla c(t, x)\right)-b K_{c} \frac{c}{\beta_{c}+c} \frac{o}{k_{o}+o} \\
\gamma_{o} o_{t}(t, x)+\nabla \cdot(v(x) o(t, x)) & =\nabla \cdot\left(D_{o}(v(x)) \nabla o(t, x)\right)-b K_{o} \frac{c}{\beta_{c}+c} \frac{o}{k_{o}+o} \\
b_{t} & =b K_{b} \frac{c}{\beta_{c}+c} \frac{o}{k_{o}+o}-d b+B .
\end{aligned}
$$

To set up an abstract formulation, we define $V=H^{1}(\Omega) \times H^{1}(\Omega) \times L^{2}(\Omega)$, and $H=$ 
$L^{2}(\Omega)^{3}$. The form $\sigma(q)$ is given by

$$
\begin{aligned}
\sigma(q)((c, o, b),(\phi, \psi, \xi)) & =\int_{\Omega}\left(D_{c}(v) \nabla c \cdot \nabla \phi-v c \cdot \nabla \phi\right) d x+\int_{\Omega}\left(D_{o}(v) \nabla o \cdot \nabla \psi-v o \cdot \nabla \psi\right) d x \\
& +\int_{\Omega} d b \xi d x,
\end{aligned}
$$

and the form $b(q)$ is defined as above. Note that the coercivity of the form $\sigma$ is easily verified under the condition that the bacteria death rate function $d$ is bounded below by a positive number (note that for the bacteria component, coercivity is with respect to $L^{2}$ ). We also remark that some models include transport of the bacteria (i.e., the bacteria are in the water, as opposed to the soil). We note that in this problem, as opposed to the "contaminant-only" model, we have at best a locally Lipschitz nonlinearity.

We return now to the inverse problem, which is the topic of the next section.

\section{Parameter Identification.}

We recall the possible collections of observations

$$
\begin{aligned}
\hat{C}_{i j}^{d} & \approx \int_{\Omega_{j}} c\left(t_{i}, x\right) d x, \\
\hat{C}_{i j}^{t} & \approx \int_{\Omega_{j}}\left(\gamma(x) c\left(t_{i}, x\right) d x,\right. \\
\hat{c}^{d}\left(t_{i}, x\right) & \approx c\left(t_{i}, x\right) \\
\hat{c}^{t}\left(t_{i}, x\right) & \approx \gamma(x) c\left(t_{i}, x\right),
\end{aligned}
$$

where $n$ denotes the number of times at which observations are made, and $m$ (in the first two cases) denotes the number of spatial samples taken per observation time. Each of these can be modeled using a continuous map from the state space $L^{2}(\Omega)$ to either $\mathbb{R}^{m n}$ (in the case of the discrete measurments) or $\left(L^{2}\right)^{n}$ (for the distributed measurements). The least squares formulation of the inverse problem is embodied in the functional

$$
J\left(q, c_{0}\right)=\left\|z-\mathcal{B} c\left(q, c_{0}\right)\right\|_{Z}^{2},
$$


to be minimized over $Q \times \mathcal{C}$. Here the space $Z$ is either $\mathbb{R}^{m n}$ or $\left(L^{2}\right)^{n}$ and the observation operator $\mathcal{B} \in \mathcal{L}(H, Z)$ is defined as in one of the observation processes above.

In order to compute minimizers, we must make some approximations. We first consider an approximation $c^{N}$ of the state variable $c$, as discussed in the previous section, leading to a cost functional

$$
J^{N}\left(q, c_{0}\right)=\left\|z-\mathcal{B} c^{N}\right\|_{Z}^{2}
$$

which is sufficiently general for all of the above mentioned measurement types. The convergence results of the previous sections guarantee that if $q^{N} \rightarrow q$ and $c_{0}^{N} \rightarrow c_{0}$, then $J^{N}\left(q^{N}, c_{0}^{N}\right) \rightarrow J\left(q, c_{0}\right)$, which will give us (see below) subsequential convergence of minimizers, if we restrict the initial conditions to a compact subset of $H=L^{2}$. Toward that end, we set

$$
\mathcal{C}_{0}=\left\{c_{0} \in H^{1}(\Omega):\left|C_{0}\right|_{H^{1}} \leq K_{7}\right\}
$$

and we let

$$
Q_{a d}=\mathcal{V} \times \mathcal{T} \times \mathcal{A} \times \mathcal{F} \times \mathcal{C}_{0}
$$

denote the space of functions to be estimated.

To compute minimizers, we must consider finite dimensional approximations to the parameter space. If $\mathcal{S} \subset C^{0,1}(\bar{\Omega})$ and if the functions in $\mathcal{S}$ are uniformly bounded, we may define a sequence $\mathcal{S}^{N}$ of piecewise linear continuous functions, satisfying the same bounds as $\mathcal{S}$. These $\mathcal{S}^{N}$ sets have the following properties.

(PA1) $\quad \forall s \in \mathcal{S}, \exists s^{N} \in \mathcal{S}^{N} \ni \sup _{\Omega}\left|s^{N}-s\right| \rightarrow 0$, and

(PA2) $\quad \forall\left\langle s^{N}\right\rangle$ sequence $\ni s^{N} \in \mathcal{S}^{N}, \exists$ autoseq $\left\langle s^{N_{k}}\right\rangle_{k=1}^{\infty}$ and $s \in \mathcal{S}^{\prime} \ni \sup _{\Omega} \mid s-$ $s^{N_{k}} \mid \rightarrow 0$. 
If $\mathcal{S} \subset L^{2}(\Omega)$, the above properties also hold when the $C$ norm is replaced with the $L^{2}$ norm.

Based on this idea, we set $Q_{a d}^{N}=\left\{\mathcal{V}^{N}\right\} \times \mathcal{T}^{N} \times \mathcal{A}^{N} \times \mathcal{F}^{N} \times \mathcal{C}_{0}^{N}$. Coupling Theorem 2 with (PA1) and (PA2), we have the following theorem.

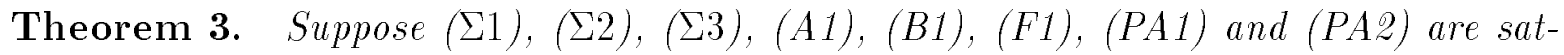
isfied. Then $\forall N$ there exists a minimizer of $J^{N}$ over $Q_{a d}^{N}$, and there exists a minimizer of $J$ over $Q_{a d}$. Moreover, minimizers of $J^{N}$ converge subsequentially to minimizers of $Q_{a d}$

Proof. The proof of this results follows directly from the abstract results of [3, pp. 142-144].

6. Some Concluding Remarks. We have presented here a theoretical framework that guides us in the design of estimation algorithms for contaminant transport problems. The framework is sufficiently general to allow several types of problems to be analyzed. Of particular importance is the determination of parameter sets which are compact and whose topologies are strong enough to provide continuous dependence.

We have made some initial computational studies, and the results, which will appear elsewhere, are promising. Our future efforts will be focused on continued modeling and on efficient computational implementation of the ideas presented above. There are a wide variety of models in the mathematical, scientific, and engineering literature (as evidenced in the references below). The research herein gives a theoretical basis for comparing models by fitting them to data. The MADE-2 experimental data (see [5]) provides an excellent testbed for comparing models in a real field scale situation. We 
are in the process of developing computational algorithms to apply our techniques to this dataset.

\section{References}

[1] H.T. Banks, "Modeling and Control in the Biomedical Sciences," Lecture Notes in Biomathematics 6, Springer-Verlag, Berlin, 1975.

[2] H.T. Banks and K. Ito, A unified framework for approximation in inverse problems for distributed parameter systems, Control: Theory and Advanced Technology 4(1) (1988), 73-90.

[3] H.T. Banks and K. Kunisch, "Estimation Techniques for Distributed Parameter Systems," Birkhäuser, Boston, 1989.

[4] J. Bear, "Dynamics of Fluids in Porous Media," Elsevier, New York, 1972.

[5] J. M. Boggs, L. M. Beard, S. E. Long, M. P. McGee, W. G. MacIntyre, C. P. Antworth, and T. B. Stauffer, Database for the Second Macrodispersion Experiment (MADE-2), Electric Power Research Institute Technical Report TR-102072, EPRI, Palo Alto, February, 1993.

[6] R. C. Borden and P. B. Bedient, Transport of Dissolved Hydrocarbons Influenced by Oxygen-Limited Biodegradation, 1: Theoretical Development, Water Resources Research 22 (13) (1986), pp. 1973-1982.

[7] R. C. Borden and P. B. Bedient, M. Lee, C. Ward, and J. Wilson, Transport of Dissolved Hydrocarbons Influenced by Oxygen-Limited Biodegradation, 2: Field Application, Water Resources Research 22 (13) (1986), pp. 1983-1990. 
[8] C. Canuto and A. Quateroni, Approximation Results for Orthogonal Polynomials in Sobolev Spaces, Mathematics of Computation, 38 (157) (1982), pp. 67-86.

[9] M. Celia, J. S. Kindred, and I. Herrera, Contaminant Transport and Biodegradation, 1: A Numerical Model for Reactive Transport, Water Resources Research 25 (6) (1989), pp. 1141-1148.

[10] C. Chiang, C. Dawson, M. F. Wheeler, Modeling of In Situ Biorestoration of Organic Compounds in Groundwater, Transport in Porous Media, 6 (1991), pp. $667-702$.

[11] N. A. Cressie, Statistics for Spatial Data, Wiley, New York, 1991.

[12] R. A. Freeze and J. Cherry, Groundwater, Prentice-Hall Englewood Cliffs, 1979.

[13] S. Gutman, Identification of Discontinuous Parameters in Flow Equations, SIAM Journal of Control and Optimization 28 (5) (1990), pp. 1049-1060.

[14] C. Johnson, Numerical Solution of Partial Differential Equatiosn by the Finite Element Method, Cambridge Press, Cambridge, 1987.

[15] J. S. Kindred and M. Celia, Contaminant Transport and Biodegradation, 2: Conceptual Model and Test Simulations, Water Resources Research 25 (6) (1989), pp. 1149-1159.

[16] K. Kunisch and L. White, Parameter Estimation for Elliptic Equations in Multidimensional Domains with Point and Flux Observations, Nonlinear Analysis, Theory, Methods and Applications, 10 (1986), pp. 121-146. 
[17] P. K. Lamm, C. K. Lo, and I. G. Rosen, Identification of Degenerate Distributed Parameter Systems, in Proc. 5th IFAC Symposium on Control of Distributed Parameter Systems, Perpignan, France, 1989.

[18] P. K. Lamm and I. G. Rosen, An Approximation Theory for the Estimation of Parameters in Degenerate Cauchy Problems, to appear.

[19] K. T. B. MacQuarrie and E. Sudicky, Simulation of Biodegradable Organic Contaminants in Groundwater, 2: Plume Behavior in Uniform and Random Flow Fields, Water Resources Research 26 (2) (1990), pp. 223-239.

[20] D. Malone, C-M. Kao, and R. C. Borden, Dissolution and Biorestoration of Nonaqueous Phase Hydrocarbons: Model Development and Laboratory Evaluation, Water Resources Research 29 (7) (1993), pp. 2203-2213.

[21] R. H. Martin, Nonlinear Operators and Differential Equations in Banach Spaces Wiley Interscience, New York, 1976.

[22] J. D. Murray, Mathematical Biology, Springer-Verlag, Berlin, 1989.

[23] R. O'Neill, D. DeAngelis, J. Pastor, B. Jackson, and W. Post, Multiple Nutrient Limitations in Ecological Models, Ecological Modelling, 46 (1989), pp. 147-163.

[24] A. Pazy, "Semigroups of Linear Operators and Applications to Partial Differential Equations," Springer-Verlag, New York, 1983.

[25] D. Piskiewicz, "Kinetics of Chemical and Enzyme-Catalyzed Reactions," Oxford, New York, 1977. 
[26] R.E. Showalter, "Hilbert Space Methods for Partial Differential Equations," Pitman Publishing Ltd., London, 1977. 